\title{
Reliability issues for a bolometer detector for ITER at high operating temperatures*
}

\author{
H. Meister, ${ }^{1, \dagger}$ M. Kannamüller, ${ }^{1}$ J. Koll, ${ }^{1}$ A. Pathak, ${ }^{1}$ F. Penzel, ${ }^{1}$ T. Trautmann, ${ }^{1}$ P. Detemple, ${ }^{2}$ S. Schmitt, ${ }^{2}$ and H. Langer ${ }^{3}$ \\ ${ }^{1}$ Max-Planck-Institut für Plasmaphysik, EURATOM Association, Boltzmannstr. 2, D-85748 Garching, Germany \\ ${ }^{2}$ Institut für Mikrotechnik Mainz, GmbH, Mainz, Germany \\ ${ }^{3}$ KRP-Mechatec Engineering GbR, Garching, Germany
}

\begin{abstract}
The first detector prototypes for the ITER bolometer diagnostic featuring a $12.5 \mu \mathrm{m}$ thick Pt-absorber have been realized and characterized in laboratory tests. The results show linear dependencies of the calibration parameters and are in line with measurements of prototypes with thinner absorbers. However, thermal cycling tests up to $450^{\circ} \mathrm{C}$ of the prototypes with thick absorbers demonstrated, that their reliability at these elevated operating temperatures is not yet sufficient. Profilometer measurements showed a deflection of the membrane hinting to stresses due to the deposition processes of the absorber. Finite element analysis (FEA) managed to reproduce the deflection and identified the highest stresses in the membrane in the region around the corners of the absorber. FEA was further used to identify changes in the geometry of the absorber with a positive impact on the intrinsic stresses of the membrane. However, further improvements are still necessary.
\end{abstract}

\section{INTRODUCTION}

The total radiated power as well as the radiation emission profile on ITER will be determined by the bolometer diagnostic. The reference detector type chosen for ITER is the metal resistor bolometer ${ }^{1}$. Due to the high neutron irradiation levels projected for ITER as well as the high operating temperatures of the surrounding structures, new materials for the bolometer detectors have to be tested and qualified. Currently, the detector development for ITER focusses on Pt absorbers on $\mathrm{SiN}$ membranes.

The first prototypes developed featured a $4.5 \mu \mathrm{m}$ thick Pt absorber on a $1.5 \mu \mathrm{m}$ thin $\mathrm{Si}_{3} \mathrm{~N}_{4}$ membrane. Several samples of these prototypes have been operated successfully in the tokamak ASDEX Upgrade since 2009. New prototypes featuring a Pt absorber of $12.5 \mu \mathrm{m}$ thickness on a $3 \mu \mathrm{m}$ membrane are now available and have been tested in a laboratory environment at temperatures up to $450^{\circ} \mathrm{C}$. The tests and results are presented in section II.

Increasing the absorber thickness also increased the stresses in the membrane leading to reduced mechanical stability. As this issue is of major importance for the application of the detector in ITER, a detailed study was initiated to determine the cause for the stresses and to identify potential mitigation strategies. These results are presented in sections III and IV. Finally, section V concludes with an outlook on further developments required.

\section{CHARACTERISATION OF PROTOTYPE SENSORS WITH THICK ABSORBERS}

One of the most crucial developments for the final ITER bolometer diagnostic is the development of a radiation hard resistive bolometer foil with thick enough absorbers to reliably detect the total radiated power from the plasma. The

*Contributed paper, published as part of the Proceedings of the 19th Topical Conference on High-Temperature Plasma Diagnostics, Monterey, California, USA, May 2012. thickness of the absorber has to match the attenuation length for the photon energies expected in ITER (up to $25 \mathrm{keV}$ in standard scenario). According to the mass-energy attenuation coefficients for Pt provided by NIST ${ }^{2}$ and taking the density of Pt near room temperature $\left(21.4 \mathrm{~g} / \mathrm{cm}^{3}\right)$, a Pt-layer of $12.5 \mu \mathrm{m}$ thickness will capture $68 \%$ of the energy of an incoming mono-energetic beam of $25 \mathrm{keV}$ photons. This leads to a temperature increase in the absorber which can be detected by the diagnostic. In the case of $10 \mathrm{keV}$ photons, $94 \%$ of the incoming energy will be absorbed. As the density of Pt has only a weak dependence on temperature, very similar results are expected for higher operating temperatures in ITER.

The relevant parameters for characterising bolometer detectors for operation in a fusion device are the calibration parameters: meander resistance $R$, cooling time constant $\tau$, and normalised heat capacity $\kappa^{3}$. They can be determined in the test facility IBOVAC (ITERBolo vacuum test facility) at IPP which provides an environment of up to $450^{\circ} \mathrm{C}$ in vacuum. The samples were heated up to this temperature at heating rates of at most $1 \mathrm{~K} / \mathrm{min}$. The calibration constants were measured once every minute according to the procedure described in [3]. Simultaneously, the temperature of the detector was measured. The results for prototypes featuring a $12.5 \mu \mathrm{m}$ absorber on a $3 \mu \mathrm{m}$ membrane are shown in FIG. 1 .

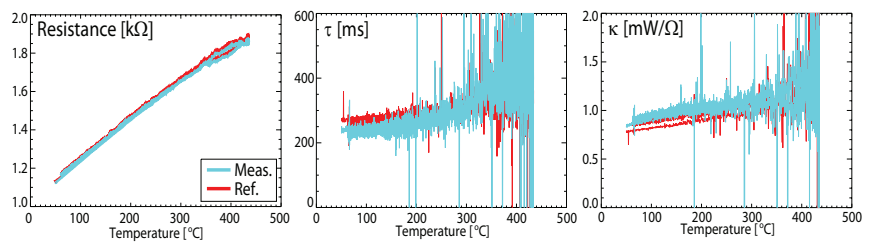

FIG. 1: (color online) Calibration parameters of a prototype bolometer detector (Flow-chart 1100025, wafer 481, chip K, channel 2) with a $12.5 \mu \mathrm{m}$ absorber for measurement (blue), and reference (red) absorber as function of temperature: $R, \tau$ and $\kappa$ (left to right).

The resistances of the meanders for both, measurement and reference absorber, show an almost linear dependence with very similar values up to $350^{\circ} \mathrm{C}$ (FIG. 1 , left). Typical temperature coefficients of resistivity (TCR) deduced from this kind of measurements in the temperature range between $20^{\circ} \mathrm{C}$ 
and $100^{\circ} \mathrm{C}$ yield values in the order of $1.8-2.1 \mathrm{mK}^{-1}$. Evaluating the linear TCR for the case shown in FIG. 1 for temperatures up to $350^{\circ} \mathrm{C}$ yields a value of $2.06 \mathrm{mK}^{-1}$. Fitting a second order polynomial instead yields the coefficients $A=$ $2.35 \cdot 10^{-3} \mathrm{~K}^{-1}$ and $B=-7.8 \cdot 10^{-7} \mathrm{~K}^{-2}$ while reducing $\chi^{2}$ by a factor of 2. This compares well to the Callendar-van-Dusen coefficients for a Pt100 resistor according to the IEC751 norm $\left(A=3.908 \cdot 10^{-3} \mathrm{~K}^{-1}\right.$ and $\left.B=-5.775 \cdot 10^{-7} \mathrm{~K}^{-2}\right)$. The differences are most likely due to the meanders being a thin film ${ }^{4}$.

Above $350^{\circ} \mathrm{C}$, a slight hysteresis between the heating and cooling phase can be seen. As in previous measurements for $4.5 \mu \mathrm{m}$ absorbers ${ }^{1}$, the hystereses depends on the sample. A closer inspection of this behaviour was performed for samples with $4.5 \mu \mathrm{m}$ thick absorbers. Despite an annealing process at $650^{\circ} \mathrm{C}$ after the deposition of the meanders, but before etching the groove for the absorber, an additional application of $450^{\circ} \mathrm{C}$ on the final prototype results in a significant change of resistance and TCR (see FIG. 2). Furthermore, depending

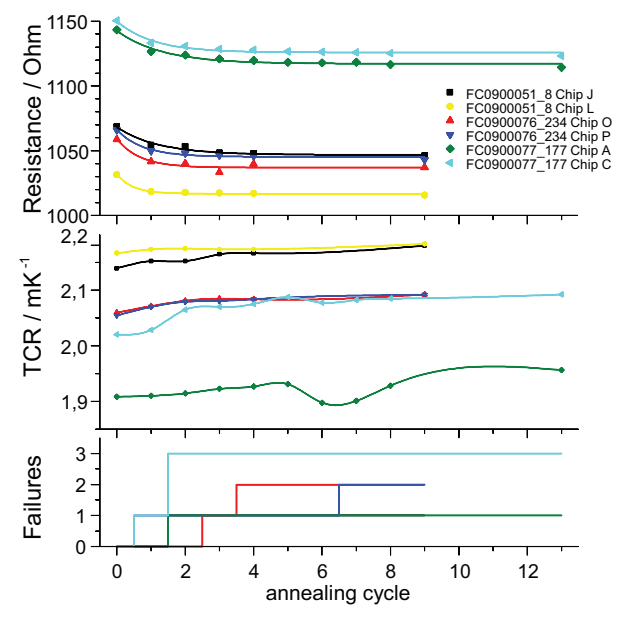

FIG. 2: (color online) Resistance, TCR and failures per 4-channeldetector (top to bottom) in dependence of the number of annealing cycles at $450^{\circ} \mathrm{C}$ applied on samples with $4.5 \mu \mathrm{m}$ absorbers.

on the sample, the membranes start to break. The breaking might happen even after completion of the annealing process at room temperature. The reason for this kind of behaviour is most probably to be found in intrinsic material stresses and the temperature dependent deflection of the membrane (see section III).

The cooling time constants $\tau$ of the new samples with $12.5 \mu \mathrm{m}$ absorber are in the range of $230-240 \mathrm{~ms}$ at room temperature. Compared to previous samples ${ }^{1}$, an additional heat conduction layer of $150 \mathrm{~nm} \mathrm{Al}$ (thermal conductivity of $\mathrm{Al}$ is three times higher than the one of $\mathrm{Pt}$ ) was added between absorber and silicon-frame of the detector. This helped to reduce the values of $\tau$ to a range which is comparable to those of detectors currently in use on devices like ASDEX Upgrade or JET. For detectors with a $4.5 \mu \mathrm{m}$ thick absorber, $\tau=175 \mathrm{~ms}$ has been achieved.

The variation of $\tau$ with temperature is usually slightly positive (FIG. 1, centre); the variation depends on the sample. The noise of the measurement increases with temperature. The reason is, that $\tau$ is determined by applying an ohmic heating pulse with a constant bridge-voltage of $3.75 \mathrm{~V}$ on the measurement and reference meanders, respectively, and fitting an exponential to the decay of the bridge current. The higher the operating temperature is, the higher is the resistance of the meanders and the lower the current to be measured.

In FIG. 1, right, the normalized heat capacity $\kappa$ is plotted for one sample as a function of temperature. A clear linear dependence can be observed. Again, the noise of the measurement increases with operating temperature for similar reasons as in the case of $\tau$. In comparison with samples featuring an absorber of $4.5 \mu \mathrm{m}, \kappa$ is higher by a factor of almost 3 as could be expected from the increase in thickness.

\section{INVESTIGATION OF FAILURE CAUSES}

As shown in section II the prototype detectors fail after a few annealing cycles at high temperatures. Additionally, calibration measurements for prototype detectors of various absorber and membrane thicknesses at elevated temperatures have shown that the increased failing of the membranes sets in when the detectors are exposed more than once to temperatures above $200^{\circ} \mathrm{C}$ (without determining the exact value). This behaviour can not be tolerated for the application in ITER and was thus investigated in more detail. A 3D-scan of the detector surface using the measurements of a profilometer showed that the membrane is deflected out of plane by $15 \mu \mathrm{m}$ (FIG. 3) with strong gradients in the region close to the edge of the absorber. In order to understand this behaviour, a finite element
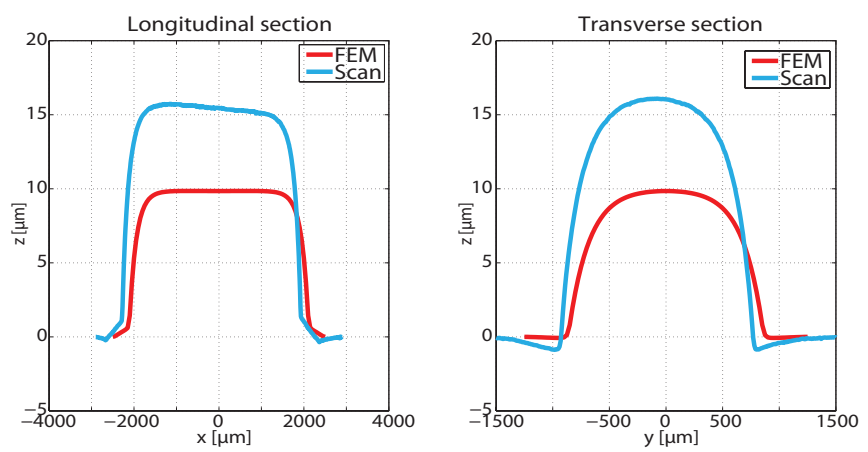

FIG. 3: (color online) Longitudinal and transverse section of a 3D scan of the surface of a detector membrane by a profilometer (blue) and the result of a non-linear FEA (red).

(FE) model has been developed for the detector membrane. Non-linear FE simulations using ANSYS 12 taking typical material properties and the initial intrinsic material stresses due to the production processes (500 MPa in $\mathrm{Pt}$ and $130 \mathrm{MPa}$ in $\mathrm{SiN}$, as measured on test samples) into account, managed to reproduce this behaviour (red curve in FIG. 3). Though the amplitude is not matched in this relative simple simulation, the shape is. This allows for relative comparisons when applying the model to different temperatures and geometrical variations of the absorber.

The behaviour of an absorber at $450^{\circ} \mathrm{C}$ was investigated us- 

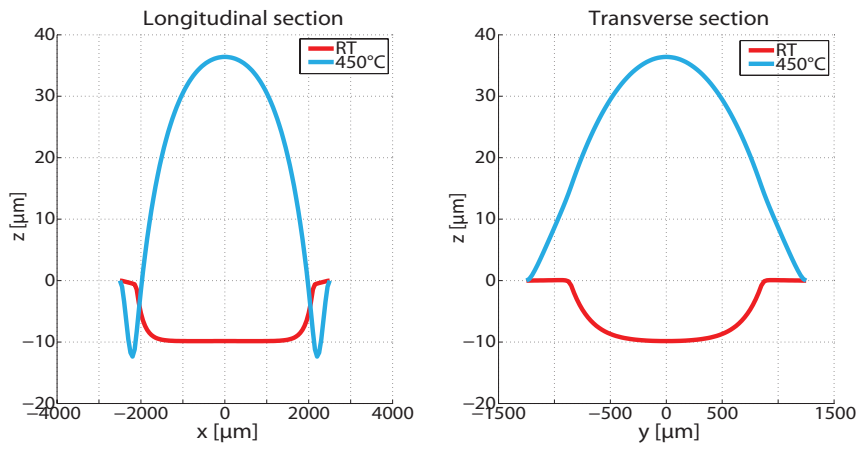

FIG. 4: (color online) Longitudinal and transverse section of a detector membrane at room temperature (red) and at $450^{\circ} \mathrm{C}$ (blue) as calculated using non-linear FEA.

ing non-linear FEA. As shown in FIG. 4, the deflection of the membrane is now in the other direction. Accordingly, subjecting a detector to annealing cycles at high temperatures results in a cyclic bending fatigue load case for the membrane due to its curvature reversal close to the absorber edge between room temperature and $450^{\circ} \mathrm{C}$. The temperature for which no membrane deflection is expected, is $\approx 270{ }^{\circ} \mathrm{C}$.

\section{ALTERNATIVE DETECTOR DESIGNS}

The non-linear FE model was used to calculate the material stresses expected for alternative absorber designs. For the reference design ${ }^{5,6}$, the highest stresses were observed in the membrane close to the corners of the absorber. From geometrical considerations, an increased corner radius should help to reduce the imposed stress. However, the FEA predicts a reduction by only $\approx 5 \%$, even for increasing the corner radius up to the point where the absorber takes an elliptical shape.

A stronger reduction in the material stresses by $\approx 35 \%$ is predicted for a "stepped" absorber. For this shape first a layer of $1 \mu \mathrm{m}$ Pt is deposited on an area which is $0.2 \mathrm{~mm}$ wider and $0.2 \mathrm{~mm}$ longer than the original absorber. Afterwards, the remaining $11.5 \mu \mathrm{m} \mathrm{Pt}$ are deposited on the original surface.

Several samples of prototype detectors have been manufactured incorporating these geometrical changes. While the production of absorbers with rounded corners was unproblematic, the results for the stepped absorber were unsatisfactory due to Pt crawling under the resist while depositing the second, thick layer. In order to improve the adhesion of the resist on the first Pt layer, further development processes are necessary. Therefore, only the behaviour of the prototypes with rounded corners could be investigated at high temperatures. Additionally, some prototypes with larger absorber, namely only the first layer of the stepped absorber, have been tested.

As could be expected from the small changes in material stress predicted by FEA, no improvement in the mechanical stability of the membranes was seen; the larger absorber exhibited an increased failure rate. The latter result suggests that a smaller absorber should be more stable.

\section{CONCLUSION}

The results presented show that the development of a new prototype bolometer resulted in a sensitive and reliable detector for the application in low to moderate ambient temperatures. At temperatures above $200^{\circ} \mathrm{C}$, the mechanical stability is insufficient. Measurements and the application of a non-linear FEA suggest that the cause for the failure is a fatigue cycle due to a reversal of the deflection direction of the membranes between room temperature and $450^{\circ} \mathrm{C}$ in combination with high stress concentration close to the absorber corners. The first prototypes incorporating geometrical changes for the reduction of the material stresses have been produced and tested. However, the desired improvement in mechanical stability could not yet be observed; a prototype with an increased absorber size showed an enhanced failure rate. In reverse conclusion this suggests that absorbers with reduced sizes could be mechanically more stable. The further detector development will have to focus on the investigation of various absorber geometries and sizes to identify the solution with the best mechanical stability for ITER.

\section{Acknowledgments}

The work presented herein has been funded by the German Federal Ministry of Education and Research (grant 03FUS0006). The sole responsibility for the contents of this publication lies with the author. $\dagger$ Electronic address: meister@ipp.mpg.de

${ }^{1}$ H. Meister, L. Giannone, L.D. Horton, G. Raupp, W. Zeidner, G. Grunda, S. Kalvin, U. Fischer, R. Reichle, A. Serikov, and S. Stickel. The ITER bolometer diagnostic - status and plans. Review of Scientific Instruments, 79:10F511-1 - 10F511-5, 2008.

2 National Institute of Standards and Technology (NIST). X-ray mass attenuation coefficients. http://physics.nist.gov/PhysRefData/XrayMassCoef/tab3.html.

${ }^{3}$ L. Giannone, K. F. Mast, M. Schubert, NBI Team, ECRH Team, and W7-AS Team. Derivation of bolometer equations relevant to operation in fusion experiments. Review of Scientific Instruments, 73(9):3205-3214, 2002.
${ }^{4}$ L. Giannone, D. Queen, F. Hellman, and J. C. Fuchs. Prototype of a radiation hard resistive bolometer for ITER. Plasma Physics and Controlled Fusion, 47:2123-2143, 2005.

5 H. Meister, T. Eich, N. Endstrasser, L. Giannone, M. Kannamüller, A. Kling, J. Koll, T. Trautmann, P. Detemple, and S. Schmitt. Optimization of a bolometer detector for ITER based on Pt absorber on SiN membrane. Review of Scientific Instruments, 81:10E132, 2010.

${ }^{6}$ K. F. Mast, J. C. Vallet, C. Andelfinger, P. Betzler, H. Kraus, and G. Schramm. A low noise highly integrated bolometer array for absolute measurement of VUV and soft-X radiation. Review of Scientific Instruments, 62(3):744-750, 1991. 